\title{
Convolutional Neural Networks to Classify Oil, Water and Gas Wells Fluid Using Acoustic Signals
}

\author{
Nafiseh Vahabi, Member IEEE and David R.Selviah, Member IEEE \\ Department of Electronic and Electrical Engineering, \\ University College London, UK, \\ e-mail: (Nafiseh.Vahabi.14@ucl.ac.uk), (Selviah.d@ucl.ac.uk).
}

\begin{abstract}
Identifying the fluid type and predicting the amount of each fluid in the fluid mixture within the well pipes are important for oil and gas production energy industry and borehole water supply. Therefore automating this process will be very valuable for the oil industry because it maximises the quality and quantity of extracted oil and reduces the cost. The current study contributes to our knowledge by addressing this important issue using machine learning algorithms. The presented paper investigates the classification algorithms that identify the fluid type in oil, water and gas pipes using acoustic signals. The datasets analysed in this study are collected from real oil, water and gas well pipes under the sea where there is no controlled environment and data contains lots of noisy signals due to unpredicted events under the sea. Data is recorded during 24 hours from Distributed Acoustic Sensors which is attached alongside the $3500 \mathrm{~m}$ of three well pipes: oil, water and gas. The acoustic dataset are in time-distance domain and are converted to frequency-wave number domain using $2 D$ fast Fourier transform. The outcome of $2 \mathrm{D}$ fast Fourier transform is sampled and fed into Artificial Neural Networks and Conventional Neural Networks algorithms to classify each fluid type. Both algorithms are trained on three datasets (oil, gas and water) and tested on another dataset. The result of this study shows Artificial Neural Networks and Conventional Neural Networks algorithms classify the fluid type with the accuracy of $79.5 \%$ and $99.3 \%$ respectively when applied on the test dataset.
\end{abstract}

Index Terms-Convolutional Neural Networks, Artificial Neural Networks, Fluid Flow Classification, Signal Processing, Distributed Acoustic Sensor

\section{INTRODUCTION}

The increase in the number of sensors used in daily life has created great volumes of data which requires storage, heavy computation and complex analysis to fully unlock the value in data. Recent advances and novel concepts in machine leaning algorithms have attracted substantial attention from industry, government, academia and health organisations, leading to the development of many novel applications to tackle the real world phenomenons. Oil and gas is one of the industries which has adapted the latest sensor technologies to automate the monitoring of flow behaviour in pipelines with the aim of improving the quality and quantity of extracted oil and reducing the cost. Despite using the latest sensors, a number of failures are still reported by oil and gas companies, such as; unknown leakage in the well, miscommunication between control room and Inflow Control Devices that are embedded in the wells under the sea. One of the main reasons is the limited studies that have been performed to analyse and interpret the data collected from the sensors. More specifically, there is a need to design and develop an alternative method to gain insight into the information extracted from the data. This insight can be provided by implementing machine learning algorithms and investigating its potential to tackle the current problem.

Downhole oil and gas pipe fluid flow classification is an increasingly important area in the oil industry and is part of the Downhole Fluid Analysis (DFA) which provides well logging and reservoir evaluation. Conventional DFA is performed by measuring one or more properties of fluid such as pressure, volume, density [1], Reynolds number and temperature [2] using corresponding sensors [3]. Identifying in-well flow regime became an important component in monitoring the oil pipes and it results in low cost intervention [4], optimising and maximising the oil production [5].

In last decade, the optical fibre sensors have been successfully developed and applied for flow measurement [5], [6], [7]. Optical fibre sensors for flow analysis in wells can do a single point measurement or multiple point measurements along the well. Traditional optical fibre sensors provided a single point measurement along the well [8], [9]. In the single point measurement, before laying out the well under the ground or sea, optical fibre is wrapped around and down the well very tightly and closely such as 10 wraps. In other sections of the well, optical fibre is wrapped slightly looser [10]. In this method the measurement occurs only at one or two points and there is no information available about the events occurring along the rest of the well. Therefore, if there is any fault such as a broken Inflow Control Valve (ICV) along the pipe, it cannot be detected. Furthermore, there is no measurements of the amount of gas or oil coming out of each ICV along the pipe because the sensor just provides a measurement at one point [11].

Recently we have seen a growing trend towards using machine learning algorithms in a variety of industrial applications. Artificial Neural Networks (ANN) are amongst the first types of machine learning algorithm that have been employed to improve the precision of ultrasonic devices and also to automate the process of fluid flow measurement in multiphase flow [12]. The combination of ANN and dual energy fan-beam gamma-ray attenuation technique improved the accuracy of oil, water and gas classification by approximately $5.68 \%$ using radial basis function for ANN training [13]. 
Identifying the pattern in gamma-ray pulse height distributions was another approach that used ANNs [14]. Scientists trained the parameters of ANNs with algorithms such as LevenbergMarquardt [15] to describe the details of two phase flow [16] and to develop a new multiphase flow metering device for real time multiphase flow classification [15]. This new device is based on training the parameters in physical models of multiphase fluid. In another study, 199 experimental data sets fed into three-layer back-propagation neural networks and achieved $97 \%$ accuracy in its prediction of flow regime [17].

Most studies in the field of multi-phase flow classification have mainly focused on modifying the structure and parameters of Artificial Neural Networks [18] to identify the pattern of each flow regime and have not dealt with processing the big data which is produced by their new developed sensors [15], [19]. In addition, they mainly used data that is collected from the flow loop in the laboratory environment.

In this study we analysed real data which contained a lot of noise as a result of unpredicted event under the sea. The unpredicted events have an impact on recorded audio signal by Distributed Acoustic Sensors. Our data was collected from Distributed Acoustic Sensors (DAS) and its application is for monitoring fluid flow, based on the speed of sound and Doppler shift measurements. Acoustic signals were preprocessed before being fed into two classification algorithms, $\mathrm{ANN}$ and CNN. Therefore, the purpose of this paper is to identify and validate the potential of classic and modern classification algorithms that predict the fluid type using real world acoustic dataset. We compared our result with the result of an experiment that used flow loop dataset in the laboratory environment. It should be noted that the acoustic datasets collected from the flow loop set up in the laboratory [20] are less noisy because they are collected in a controlled environment with low pressure. Our dataset is from real world wells that are more than $1000 \mathrm{~m}$ long and contain noise from the unpredicted events such as animals hitting the pipe and slugs. Also, the pressure under the sea is higher than the laboratory environment, which can effect the flow turbulence in the wells and the back scattered signals from optical fibre.

The remainder of this paper is organised as follows: Section I-A describes the dataset and how Distributed Acoustic Sensor (DAS) collects acoustic data from well pipe. Section II presents the pre-processing step, implementation of the Artificial Neural Network (ANN) and also describes the structure and details of Convolutional Neural Network (CNN) algorithm to classify oil, water and gas datasets. Section III discusses the result of both the ANN and the $\mathrm{CNN}$ algorithms. The conclusion section highlights the summary of finding.

\section{A. Dataset}

Distributed Acoustic sensor (DAS) made of an optoelectronic unit and optical fibre cable. An optoelectronic unit is located at the surface of the sea [21], [5]. This is an optical fibre which is run down a well alongside a pipe carrying a fluid and it is attached to that pipe at the points with a meter apart. Then a laser pulse is sent down the optical fibre from the optoelectronic unit. The light reflects from non-uniformities in the glass from every point along the fibre and a small amount of it is naturally scattered [22]. The optoelectronic unit measure all the axial strain changes occurring through the optical fibre. As the fluid mixture travels through the pipe its motion is very turbulent and it generates a noisy sound. This sound affects the light pulse so that when it is received at the surface, the sound can be recorded from every depth as if it was an array of thousands of microphones about a metre apart all along the well pipe over a distance of thousands of metres.

The acoustic datasets are collected from three different well pipes; oil, gas well and water. The sensing fiber cable that was around $3500 \mathrm{~m}$ long strapped to the production tubing and data is collected with $10 \mathrm{kHz}$ temporal frequency. The optical fibre cables were permanently clamped and installed along the production injection tubing pipe. The pipe acts like an acoustic wave guide tending to guide sound waves up and down the pipe. The sound is recorded continuously at every meter and this results in a very large amount of data recorded daily, typically 48 Terabytes. Table I provides the details of each pipeline such as the length of the optical fibre for each pipe. The Packer True Vertical Depth is the vertical distance from a final depth to a point at the surface which is usually less than the optical fibre length. The other parameters of the pipes such as their diameter and pipe material are not considered in this study nor the acoustic wave guide properties of the well bore. In our analysis we do not use all the data and we just considered the sample of datasets that are collected from the sensors along $1000 \mathrm{~m}$ of pipe.

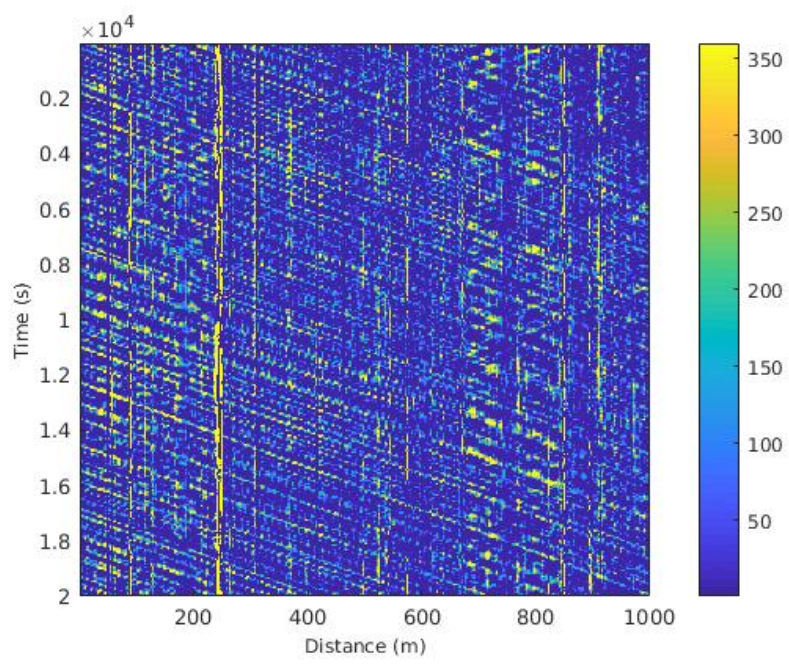

Fig. 1. Sample of acoustic raw data in time-distance domain.

\section{Method}

Two approaches are implemented to classify oil, water and gas well pipes, ANN and CNN. Acoustic dataset were preprocessed before fed into $\mathrm{ANN}$ and $\mathrm{CNN}$. Fig. 2 shows an overview of ANN and CNN that are implemented in this study. 
TABLE I

THE PROPERTIES OF OIL, GAS AND WATER WELLS

\begin{tabular}{ccc} 
Well Type & Packer True Vertical Depth (m) & Length Optical (m) \\
\hline Oil & 2600 & 3000 \\
Gas & 2700 & 3500 \\
Water & 2400 & 3200
\end{tabular}

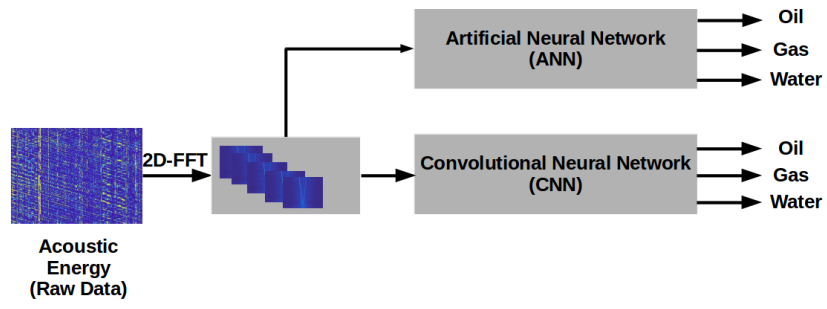

Fig. 2. Overview diagram for classifying fluid flow regime in oil, gas and water well pipes. Acoustic data are converted from time-domain to frequencywave number domain using 2D-FFT.

\section{A. Pre-processing Acoustic Dataset}

Two dimensional fast Fourier transform is defined by $F(k, \omega)$ (Eq. 1) where:

$$
F(k, \omega)=\iint f(x, t) e^{-2 \pi i(k x-\omega t)} \mathrm{dxdt}
$$

Where $k$ and $\omega$ represent the wave number and frequency of the sample data respectively. In order to increase the performance of the 2D FFT algorithm, we should transform the height and width of an image to an integer power of two [23]. Hence, a two dimensional fast Fourier transform is applied with a moving window of 256 samples in distance and 16,384 samples in time. 256 samples in distance are chosen to maintain a good number of spatial frequency bins [24]. 16,384 samples in time are chosen because of the sampling rate in time is $10 \mathrm{kHz}$ and 16,384 is the closest power of 2 number which is not sampling lower than the Nyquist frequency [25]. The result, $F(k, \omega)$, contains the V-shaped lines, as the acoustic waves travel in both directions along the fiber.

As seen in Fig. 4, the angle of V-shape in oil, gas and water wells is clearly different because of unique property of each fluid.

\section{B. Articial Neural Network}

The structure of the ANN contains 16 hidden layers with the following input data: acoustic energy of oil, water and gas dataset during 20,000 s and within $1000 \mathrm{~m}$. The data is divided into three sets: $70 \%$ for training, $15 \%$ for testing and $15 \%$ for validation. Levenberg-Marquardt (LM) is a preferred method as suggested in literature [31]. The Levenberg-Marquardt is designed to minimise the loss functions made up of a sum of squared errors [31]. Cross entropy is implemented to prevent over fitting. The number of hidden units is tested for training the network and 60 hidden units result in the least validation lost as shown in Table II.

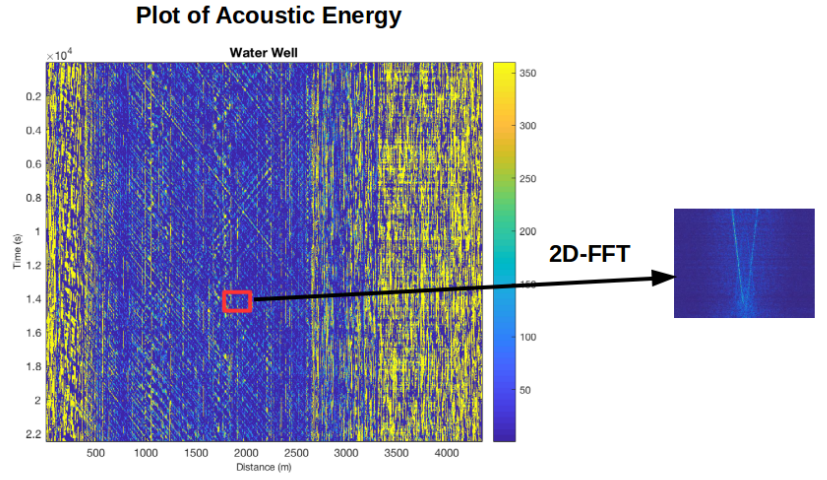

Fig. 3. A sample of V-shape image in oil dataset which obtained after applying 2D-FFT to time-distance acoustic data.

TABLE II

Number of hidDEn Units in ARTIFICIAL NEURAL NETwork.

\begin{tabular}{cc}
\hline Number of Hidden Units & Validation Loss \\
\hline 20 & 0.9751 \\
40 & 0.9151 \\
60 & 0.8881 \\
80 & 0.8937 \\
100 & 0.9018 \\
\hline
\end{tabular}

ANN takes three datasets and classifies them into oil, gas or water category. Fig. 5 presents the outcome of ANN on the training, testing and validation datasets. On the testing dataset, $79.5 \%$ of the predictions were correct and $20.5 \%$ were incorrect classifications. Fluid in water pipe is predicted with the highest accuracy, $97.8 \%$. Oil and gas fluid pipe are classified with $84.0 \%$ and $66.9 \%$ accuracy accordingly as shown in the testing confusion matrix (Fig. 5).

\section{Convolutional Neural Network}

One of the most successful types of Neural Network with a great result in a variety of pattern recognition applications is called Convolutional Neural Network (CNN) [26]. In the network with CNN structure there are many copies of the same neuron that develop a large neural network with the smaller number of parameters. Therefore, the network does not need to learn a large number of parameters and it uses one neuron in many places. This will dramatically reduce the learning error in CNN structure. In this implementation the Neural Network Tool box from Matlab $2018 b$ version library was used and its architecture is shown in Fig. 7.

Convolutional Neural Network is fed with the pre-processed data from three well pipes. In the pre-processing stage, data is transformed from time-distance domain to frequency-wave number domain using two dimensional Fast Fourier Transform (2D-FFT) algorithm. 1000 sample of V-shape images from each dataset (1000 samples from oil pipe, 1000 sample from water pipe, 1000 samples from gas pipe) fed into the input layer of the network. The Convolutional layer is formed by 


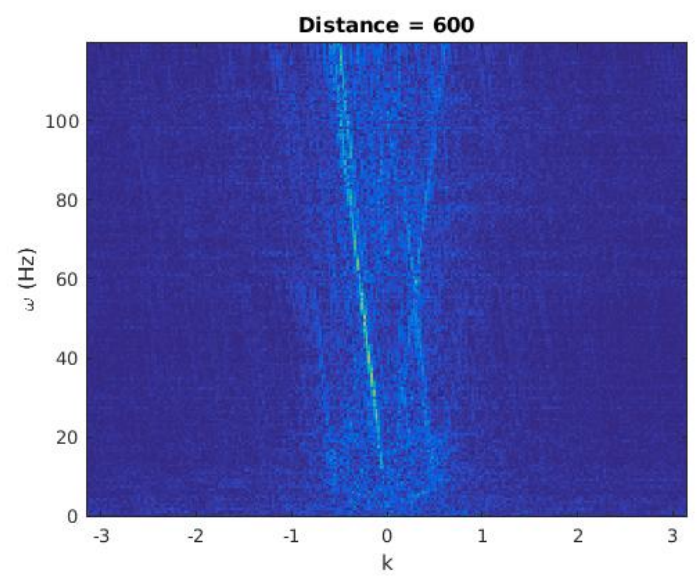

(a) water well

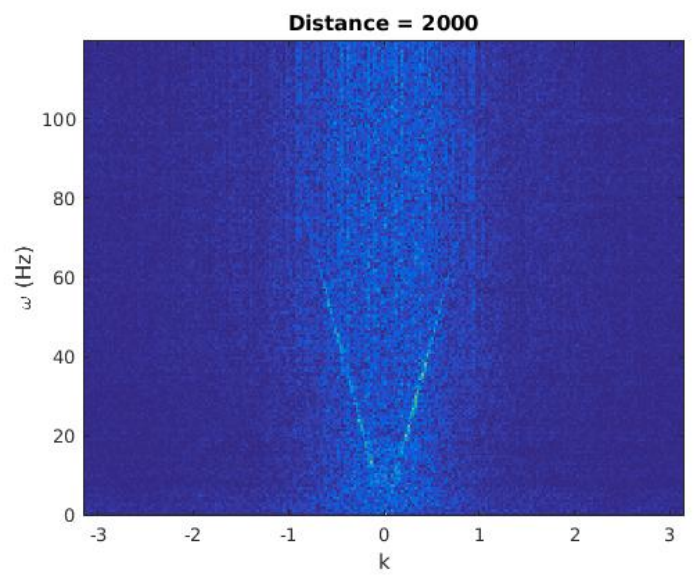

(b) Gas well

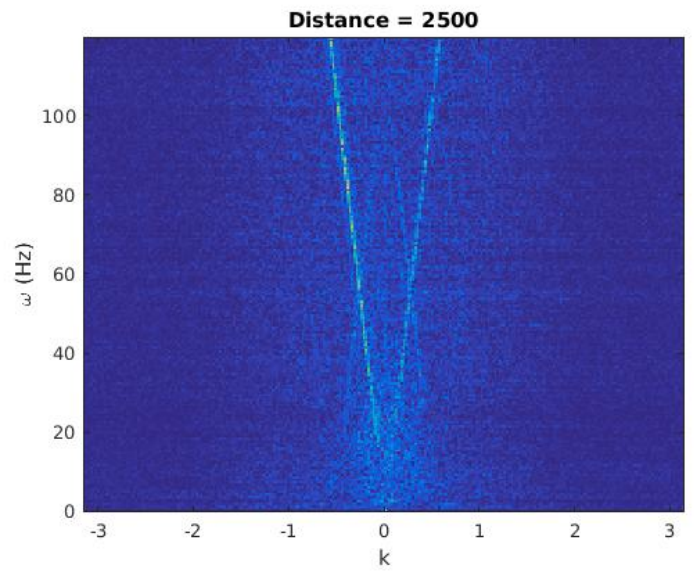

(c) Oil well

Fig. 4. A sample of the V-shape angle in each well pipe at different distance along the pipeline.

neurons that might have parallel or multilayer architecture. These neurons connect to the small regions of the input images or their previous layer. These small areas are called filters, whose size needs to be defined. For each region, a
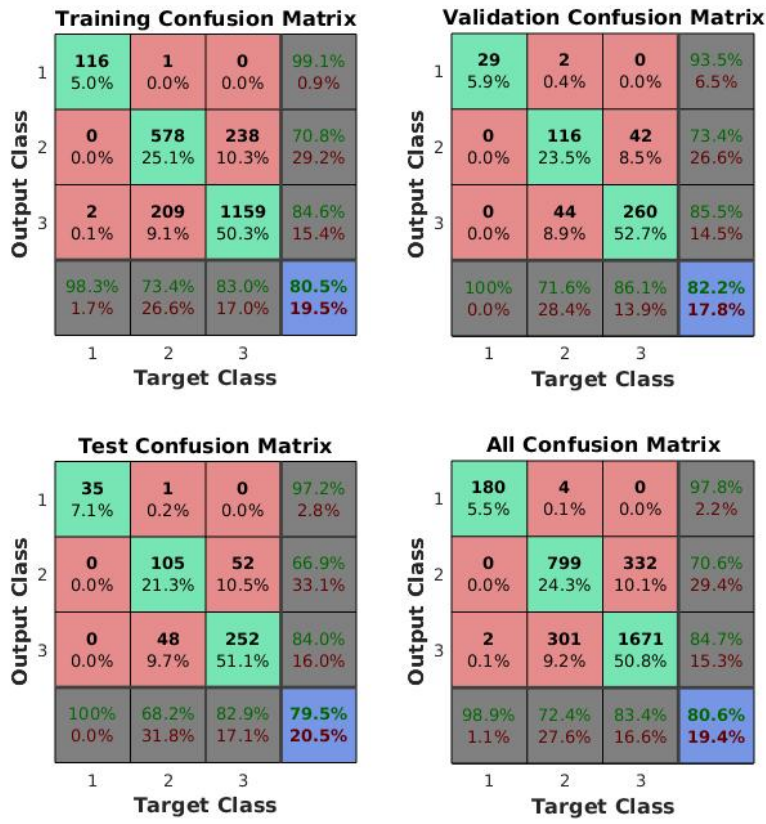

Fig. 5. Classification result for oil, water and gas dataset. The first three diagonal cells show the number and percentage of correct classifications by the trained network. Class 1,2 and 3 are corresponded to water, gas and oil datasets. Overall, the ANN classifier predict the water type with higher accuracy ( $97.2 \%$ in testing confusion matrix) than oil and gas well pipe.

basic calculation of a neural network is performed that is a dot product of the input and the weights, and then add a bias. An input image is convolved by scanning the filter both horizontally and vertically along the image and repeating the same calculation. In addition to the filter size, the step size for moving the filter needs to be specified [27]. Each filter uses the same set of weights and biases to scan the whole input image. The outcome of the convolution creates a feature map. Therefore, the number of filters determines the number of feature maps in the convolution layer [28], [29]. Fig. 8 shows some features are extracted from Convolutinal layer of the CNN network.

Rectified Linear Unit layer is also implemented where the activation function is defined to performs a threshold operation to each component of its input [30]. A max-pooling layer is used to down sample the parameters in the network. Maxpooling function takes an input from the activation function and outputs the maximum values of the rectangular area in its input. In the network with multiple convolutional layers, a max-pooling layer is required between each of the two Convolutional layers to reduce the number of parameters. All features learned by the previous layers are combined in a fully connected Layer to classify the input image. Therefore, the number of outputs of this layer equals the number of classes the images belongs to. In our dataset this number is set to three (water, oil and gas). Classification layer is the last layer of the network. 

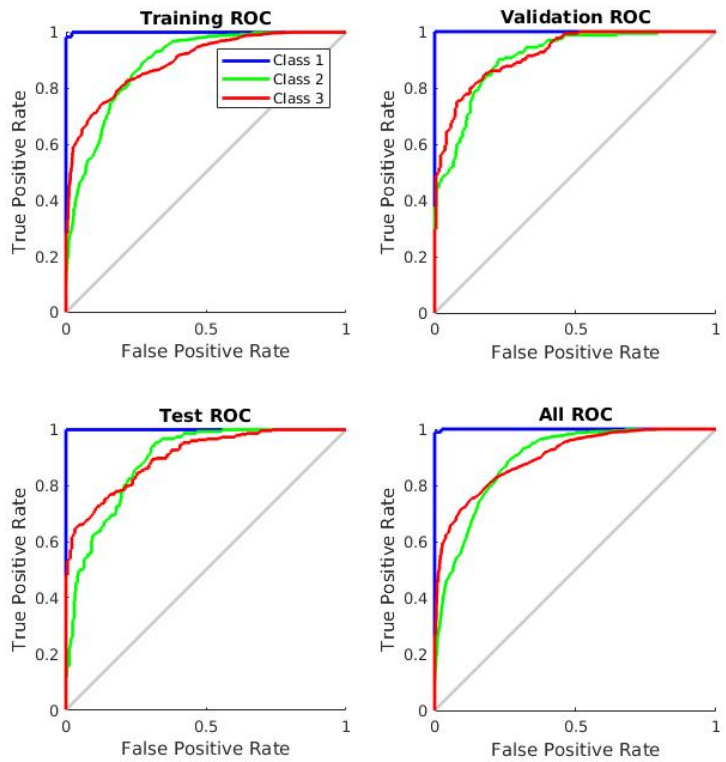

Fig. 6. Comparing the area under the curve for water (class 1), oil (class 2 ) and gas (class 3) classification in ANN algorithm. The water classification has the highest value which shows the best performance of the classifier on this dataset.

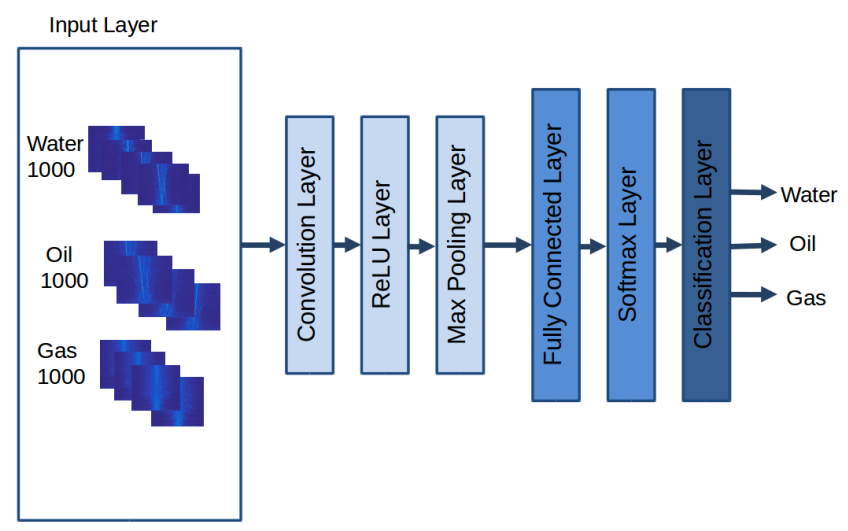

Fig. 7. Structure of Convolutional Neural Network. 1000 samples of each dataset (oil, water and gas) are fed into CNN input layer.

\section{RESULT}

The Artificial Neural Network and Convolutional neural Network algorithms were implemented successfully. Both algorithms used Matlab software version 2018 and were trained on CPU. We trained our algorithms on the dataset collected from along $1000 \mathrm{~m}$ of the well pipe. The result is validated by applying the classification algorithms on a test dataset collected from sensors on a different part of the pipe (between $1500 \mathrm{~m}$ and $2000 \mathrm{~m}$ ). The result of ANN are presented in Fig. 5. As shown in the figure, the validation error is $17.8 \%$ (Fig. 5 top right matrix), 29 samples are correctly classified as water (class 1), 116 cases are correctly classified as gas which corresponds to $23.5 \%$ of all 493 samples. The

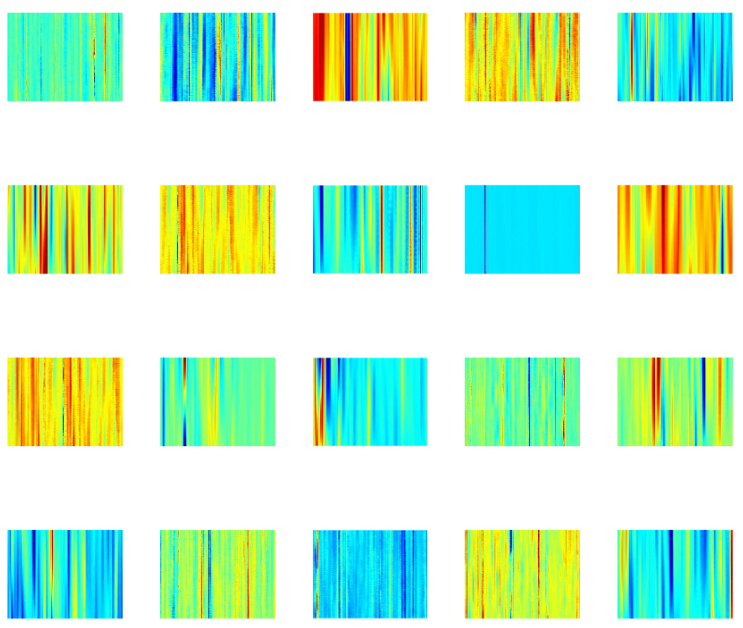

Fig. 8. Features of oil, water and gas flow regime that are extracted by deeper layers of $\mathrm{CNN}$

TABLE III

Result of Classification Algorithms

\begin{tabular}{|ccc|} 
Method & Accuracy & Time s \\
\hline ANN Training & $80.5 \%$ & 0.02 \\
ANN Testing & $79.5 \%$ & 0.001 \\
CNN Training & $100 \%$ & 3135.30 \\
CNN Testing & $99.3 \%$ & 0.01 \\
Artificial Neural Network in Ref. [20] & $100 \%$ & Not reported \\
\hline
\end{tabular}

best validation performance belongs to water dataset and the validation performance for the other two datasets, oil and gas, are very similar. Convolutional Neural Networks classified the data with higher accuracy, $99.3 \%$ on testing dataset, however the training time is greater than ANN as summarised in Table. I. The result of our classification algorithms are compared with the result of ANN used in Ref. [20] as shown in Table III. It should be noted that the dataset in Ref. [20] is collected from the laboratory and is fed into ANN with 20 hidden units. Our dataset are from real world wells which means, it contains noise from the unpredicted events under the sea that effect recorded sounds.

\section{CONCLusion}

The present study was designed to investigate and validate the algorithms used for the classification of the fluid types oil, water and gas datasets. Artificial Neural network and Convolutional neural Network algorithms were implemented for this purpose. The most obvious finding to emerge from this study is that Convolutional neural Networks outperformed ANN to classify our datasets with accuracy of $99.3 \%$. However, the training time for $\mathrm{CNN}$ is higher than ANN algorithm. The 
implications of this analysis will greatly assist in automating the fluid type prediction in the oil and gas pipe fluid mixture in the industry. Most importantly, identifying in-well flow regime is a key component in monitoring the oil pipes and it results in low cost intervention, optimising and maximising the oil production. All the analysis in this work is performed off-line and one of the most important next steps for this research will be implementing the existing algorithms online by considering the latest hardware technologies such as NVIDIA GPUs that are capable of improving the training time and efficiency of CNN.

\section{ACKNOWLEDGMENT}

The authors thank Statoil and Silixa for permitting the use of the iDAS data acquired as part of a multiple well field trial campaign for the data analysis described in this paper. The authors thank the UK Government for funding via the Technology Strategy Board, TSB, Project Number 100722, Distributed Acoustic Flow Meter with project partners Chevron, Statoil, Saudi Aramco, Silixa, UCL and Weatherford. The author Nafiseh Vahabi thanks the UK Government Research council EPSRC for funding her studentship.

\section{REFERENCES}

[1] Basumatary, T., Singh, H. K., Chetia, D., Bezboruah, T. " Fibre optic sensor based viscometer to measure viscosity of Newtonian fluids" 2017 International Conference on Innovations in Electronics, Signal Processing and Communication (IESC), 2017, 132-136.

[2] Chen, Y., Han, Q., Yan, W., Yao, Y., Liu, T. “ Magnetic field and temperature sensing based on a macro-bending fiber structure and an FBG.” IEEE Sensors Journal, 2016, 16(21): 7659-7662.

[3] Dong, C., O'Keefe, M. D., Elshahawi, H., Hashem, M., Williams, S. M., Stensland, D., Hegeman, P. S., Vasques, R. R, Terabayashi, T., Mullins, O.C. " New downhole-fluid-analysis tool for improved reservoir characterization." SPE Reservoir Evaluation \& Engineering, 2008, 11(6): $1-107$.

[4] Roshani, G., Feghhi, S., Mahmoudi-Aznaveh, A., Nazemi, E., AdinehVand, A. " Precise volume fraction prediction in oil-water-gas multiphase flows by means of gamma-ray attenuation and artificial neural networks using one detector." Journal of Measurement, 2014, 51, 34-41.

[5] Xiao, J. J., Farhadiroushan, M., Clarke, A., Abdalmohsen, R. A., Alyan, E., Parker, T. R., Shawash, J., Milne, H. C. "Intelligent Distributed Acoustic Sensing for In-well Monitoring" SPE Saudi Arabia Section Technical Symposium and Exhibition, 2014.

[6] Vahabi, N., Alabdullah, M., Selviah, D. R. "Acoustic Sensors to Measure Speed of Oil Flow in Downhole Pipes" 14th IEEE Conference on Industrial Electronics and Applications (ICIEA), 2019, 2411-2416.

[7] Zhu, J. J., Zhang, A. P., Xia, T.H., He, S., Xue, W. "Fiber-optic hightemperature sensor based on thin-core fiber modal interferometer" IEEE Sensors Journal, 2010, 10(9): 1415-1418.

[8] Thekkethil, S. R., Venkatesan, V. N., Neumann, H., Ramalingam, R., "Design of cryogenic flow meter using fiber Bragg grating sensors" IEEE SENSORS, 2015, 1-4.

[9] Chen, T., Maklad, M., Swinehart, P. R., Chen, K. P. "Self-heated optical fiber sensor array for cryogenic fluid level sensing" IEEE Sensors Journal, 2010, 11(4): 1051-1052.

[10] Ascorbe, J., Corres, J. M., Arregui, F. J., Matias, I. R. "Magnetic field sensor based on a single mode-multimode-single mode optical fiber structure" 2015 IEEE Sensors, 2015, 1-4.

[11] Ma, Y., Muilwijk, C., Yan, Y. , Zhang, X., Li, H. Xie, T., Qin, Z., Sun, W., Lewis, E. "Measurement of Bubble Flow Frequency in Chemical Processes Using an Optical Fiber Sensor" 2018 IEEE SENSORS, 2018, $1-4$.

[12] Al-Naser, M., Elshafei, M., Al-Sarkhi, A. " Artificial neural network application for multiphase flow patterns detection: A new approach." Journal of Petroleum Science and Engineering, 2016, 145, 548-564.
[13] Roshani, G., Nazemi, E. and Roshani, M. M. “ Intelligent recognition of gas-oil-water three-phase flow regime and determination of volume fraction using radial basis function." Flow Measurement and Instrumentation, 2016, 54, 39-45.

[14] Salgado, C. M., Pereira, C. M. N. A., Schirru, R. S, Brandão, L. E. B. “ Flow regime identification and volume fraction prediction in multiphase flows by means of gamma-ray attenuation and artificial neural networks" Progress in Nuclear Energy, 2010, 52(6): 555-562.

[15] Meribout, M., Al-Rawahi, N. Z., Al-Naamany, A. M., Al-Bimani, A. Al-Busaidi, K., Meribout, A. "A multisensor intelligent device for real-time multiphase flow metering in oil fields" IEEE Transactions on Instrumentation and Measurement, 2010, 59(6): 1507-1519.

[16] Inoue, E. H., Carvalho, R. D. M., Estevam, V., Bannwart, A.C., Fileti, A. M. F. "Development of a neural network for the identification of multiphase flow pattern" 14th International Conference on Intelligent Systems and Control, 2013.

[17] Osman, E. A. " Artificial neural network models for identifying flow regimes and predicting liquid holdup in horizontal multiphase flow" $S P E$ production \& facilities, 2004, 19(1): 33-40.

[18] Madani, K. "Artificial Neural Networks Based Image Processing and Pattern Recognition: From Concepts to Real-World Applications" Image Processing Theory, Tools and Applications, 2008. IPTA 2008. First Workshops on, 2008, 1-9.

[19] Vahabi, N., Selviah, D. R. “ Dimensionality Reduction and Pattern Recognition of Flow Regime Using Acoustic Data" Proceedings of SAI Intelligent Systems Conference, 2018, 880-891.

[20] Silkina, T. "Application of Distributed Acoustic Sensing to Flow Regime Classification" https://core.ac.uk/download/pdf/52098723.pdf, Accessed: 2018-08-22.

[21] Baldwin C. S. "Applications for fiber optic sensing in the upstream oil and gas industry," Fiber Optic Sensors and Applications XII, 2015, 9480, 94800D,

[22] Hicke, K., Hussels, M., Eisermann, R., Chruscicki, S., Krebber, K., "Condition monitoring of industrial infrastructures using distributed fibre optic acoustic sensors," Optical Fiber Sensors Conference (OFS), 2017 25 th, $1-4$.

[23] Ervin S., Igor D. and Ljubia S., "Fractional Fourier transform as a signal processing tool: An overview of recent developments," IEEE Signal Processing Magazine, 2011, 91(6): 1351-1369.

[24] Park, C. S., "2D Discrete Fourier Transform on Sliding Windows," IEEE Transactions on Image Processing, 2015, 24(3): 901-907.

[25] Tao, R., Li, Y. L. and Wang, Y., "Short-Time Fractional Fourier Transform and Its Applications," IEEE Transactions on Signal Processing, 2010, 58(5): 2568-2580

[26] Shin, H. C., Roth, H.R., Gao, M., Lu, L., Xu, Z., Nogues, I., Yao, J., Mollura, D., Summers, R.M. "Deep convolutional neural networks for computer-aided detection: $\mathrm{CNN}$ architectures, dataset characteristics and transfer learning" IEEE Transactions on Medical Imaging, 2016, 35(5): $1285-1298$.

[27] Lena, P. D., Nagata, K., Baldi, P. F. "Deep spatio-temporal architectures and learning for protein structure prediction" Advances in neural information processing systems, 2012, 512-520.

[28] Selviah, D.R., Shawash, J. " Fifty years of electronic hardware implementations of first and higher order neural networks" Artificial Higher Order Neural Networks for Computer Science and Engineering: Trends for Emerging Applications, 2010, 269-285.

[29] He, K., Zhang, X., Ren, S., Sun, J. "Spatial pyramid pooling in deep convolutional networks for visual recognition" IEEE transactions on pattern analysis and machine intelligence, 2015, 37(9): 1904-1916.

[30] Nair, V., Hinton, G. E. "Rectified linear units improve restricted boltzmann machines" Proceedings of the 27th international conference on machine learning (ICML-10), 2010, 807-814.

[31] Shawash, J., Selviah, D. R. " Real-time nonlinear parameter estimation using the Levenberg-Marquardt algorithm on field programmable gate arrays" IEEE Transactions on Industrial Electronics, 2013, 60(1): 170-176.

[32] Hoo-Chang, S., Roth, H. R., Gao, M., Lu, L., Xu, Z., Nogues, I., Yao, J., Mollura, D., Summers, R. M. “ Deep convolutional neural networks for computer-aided detection: $\mathrm{CNN}$ architectures, dataset characteristics and transfer learning' IEEE transactions on medical imaging, 2016, 35(5): 1285 . 